\title{
"Die unwiderstehliche Allmacht des Geschlechtstriebes" Policeygesetzgebung und sexuelle Devianz zwischen Regulierung, Kriminalisierung und Liberalisierung
}

\author{
"The Irresistible Omnipotence of the Sexual Drive" \\ Police Ordinances and Sexual Deviance between Regulation, Criminalisation and Liberalisation \\ The chapter observes the function of early modern police ordinances regarding the criminalisation of sexual deviant \\ behaviour, the legal differentiation of sexual offences and the expansion of sanctions. It identifies two periods of more \\ intense regulation in the 16 th and 18 th centuries related to the reformation, which intensified criminalisation and \\ social control, and the reforms of 'enlightened absolutism' that intended a decriminalisation as well as an increase in \\ birth rates. An exemplary analysis of criminal justice in the electorate of Mainz investigates the enforcement of the \\ ordinances and the interdependencies of 'gute Policey' and judicial practice that also provided opportunities for the \\ subjects to use criminal justice and to negotiate sexual deviance.
}

Keywords: administrative law - criminalisation - police ordinances - sexual offences

Mit der Auflösung des Heiligen Römischen Reiches Deutscher Nation im August 1806 entstanden zahlreiche souveräne deutsche Staaten, die nicht mehr an die strafrechtlichen Vorgaben des Reichsrechts gebunden waren und Verwaltungs-, Rechts- und Justizreformen durchführen konnten oder mussten. Insofern schien der Weg frei, um auch das Sexualstrafrecht zu reformieren und weiter zu entkriminalisieren, wie es der Aufklärungsdiskurs gefordert und der Napoleonische Code pénal von 1810 umgesetzt hatte. ${ }^{1}$ Zahlreiche der neuen Staaten hatten bereits als Reichsstände in der zweiten Hälfte des 18. Jahrhunderts damit begonnen, entsprechende Reformen einzuleiten, die beispielsweise in den Entwurf des neuen bayerischen Strafgesetzbu-

${ }^{1}$ Hull, Sexuality, TAEGER, Moralstrafrecht. ches einflossen. ${ }^{2}$ Dazu gehörte auch das Kurfürstentum Mainz, das zwar ebenfalls aufgelöst worden war, aber unter dem letzten Kurfürsten Carl von Dalberg in veränderter Gestalt als „Primatialstaat" und Großherzogtum Frankfurt bis 1813 weiter existierte. ${ }^{3}$ Im Juni 1808 verzeichnete die Regierung des Primatialstaats allerdings einen starken Anstieg der Sexualdelikte im Bereich des außerehelichen Geschlechtsverkehrs: Die „nichtehelichen Schwängerungen und Ehebrüche nehmen in solchem Grade zu, daß die bisherigen Gesetze nicht mehr hinreichend scheinen", so Regierungsrat Molitor, der bereits vor 1806 für die strafrechtliche Verfolgung und die policeyliche Regulierung

\footnotetext{
${ }^{2}$ Zum bayerischen Strafgesetzbuch: HÄRTER, Feuerbach.

${ }^{3}$ HÄRTER, Carl Theodor von Dalberg; HÄRTER, Policey und Strafjustiz.
} 
sexueller Devianz zuständig gewesen war. Die „unwiderstehliche Allmacht des Geschlechtstriebes" lasse aber eine Entkriminalisierung, wie sie der Entwurf für das bayerische Strafgesetzbuch nach französischem Beispiel vorgenommen habe, nicht zu. Das „Übel [...] fleischlicher Vergehungen“ könne nur „durch geschärfte PolizeiVerordnungen" eingedämmt werden. Von den niederen Gerichten verhängte Geldstrafen würden wenig „zur Bezähmung heftiger Leidenschaften" beitragen, auch wenn sie dem Mainzer Vorgängerstaat in der Vergangenheit allein für die Unzuchtsfälle jährliche Einnahmen von 800 bis 900 Gulden gebracht hätten. Daher forderte er, "alle fleischlichen Vergehungen künftig der Regel nach mit körperlichen Strafen, das ist mit Arrest, schwerer öffentlicher Arbeit, einer Tracht Schläge" durch die Polizeidirektion bestrafen zu lassen, denn alle „Präventions- und Fornikationsfälle gehören in den polizeilichen Wirkungskreis"; Ehebruch solle dagegen weiterhin von der Kriminalgerichtsbarkeit mit mindestens einem Jahr Zuchthaus bestraft werden. Das parallele Gutachten von Regierungsrat Münch argumentierte dagegen stärker für die Entkriminalisierung von Unzucht und Ehebruch, wie sie der Aufklärungsdiskurs vorgeschlagen und Frankreich realisiert habe, denn "fleischliche Vergehungen sind Befriedigungen des Geschlechtstriebes zwischen Personen, denen der Staat - nicht aber die Natur Gesetze solches verbieten“. Erziehung und Disziplinierung seien daher bessere Mittel und wo Strafen nötig seien, sollten diese allein durch zentrale Gerichte verhängt, aber auf eine Verschärfung durch neue Gesetzgebung verzichtet werden. ${ }^{4}$

Diese Kontroverse zu Beginn des 19. Jahrhunderts ist charakteristisch für das Problem der Entkriminalisierung der Sexualdelikte und ihrer

\footnotetext{
${ }^{4}$ Gutachten Regierungsrat Molitor, 3.6.1808 und Gutachten Regierungsrat Münch, 3.6.1808, Bayerisches Staatsarchiv Würzburg, Mainzer Regierungsarchiv, Centakten 160.
}

Zuordnung zum Strafrecht oder zur Policeygesetzgebung und damit in die Strafkompetenz von unterschiedlichen Justiz- und Verwaltungsorganen. ${ }^{5}$ Der um Reformen des Strafrechts kreisende Aufklärungsdiskurs und die strafrechtlichen Kodifikationsentwürfe sowie die entstehenden Strafgesetzbücher hatten diese Thematik seit dem letzten Drittel des 18. Jahrhunderts aufgegriffen, waren aber zu unterschiedlichen Ergebnissen gekommen: Sollten Sexualdelikte wie Unzucht, Ehebruch, Prostitution, Kuppelei, Inzest oder Sodomie wie im französischen Code pénal entkriminalisiert oder mit milderen Strafen im Strafrecht verbleiben und wie konnte der Strafgrund rechtstheoretisch bestimmt werden? Gehörten sie in neu zu erlassende Polizeistrafgesetzbücher oder sollten sie weiterhin der policeylichen Einzelgesetzgebung vorbehalten sein? ${ }^{6}$ Diese Gemengelage entstand nicht erst um 1800, sondern ist das Ergebnis einer Entwicklung, die im Alten Reich bereits zu Beginn der Frühen Neuzeit einsetzte: die Regulierung und Normierung von Ehe und Sexualität in der Ordnungsund Policeygesetzgebung und die daraus resultierende „Verpoliceylichung" der Sexualdelikte, die im Folgenden näher dargestellt werden soll. Sexuelle Devianz wurde im frühneuzeitlichen Alten Reich zu einem wesentlichen Bereich der "guten Ordnung und Policey" eines Gemeinwesens und wurde von einer ausufernden Ordnungs- und Policeygesetzgebung aller Obrigkeiten - Kaiser und Reich, Landesherren und Territorialstaaten, Reichsstädte und intermediäre Gewalten - reguliert und kriminalisiert. Dies betraf nicht nur „unsittliches Verhalten“, „Illegitimität", „Leichtfertigkeit" und Verstöße gegen die Eheschließungsnormen, sondern auch Kriminaldelikte wie „Unzucht“ bzw. „Fornikation“

\footnotetext{
${ }^{5}$ Zur frühneuzeitlichen Policey zusammenfassend: HäRTER, Polizei 170-180; HärTER, Artikel ,Policey 645-652.

${ }^{6}$ Vgl. Hull, Sexuality; HäRTER, Feuerbach 135-141 AMMERER, Schwert und Galgen 175f. u. 255-258.
} 
und „Prävention" (vorehelicher Geschlechtsverkehr ohne bzw. mit anschließender EheschlieBung), Ehebruch, Prostitution, Kuppelei, Bigamie, Konkubinat, Vergewaltigung, Inzest und Sodomie.7 Als "Policeysache“ hatten die Sexualdelikte allerdings keinen festen normativen Ort: Sie gehörten auch zu den Kriminalverbrechen und damit in den Bereich des peinlichen Rechts, sie waren als „Sünden“ dem religiöskirchlichen Rechtsbereich zugeordnet und wurden in Kirchen- und Eheordnungen aufgegriffen, konnten aber auch eng mit zivilrechtlichen Konflikten und Normen im Bereich von Ehe und Familie verbunden sein. ${ }^{8}$

Diese starke Ausprägung von Multinormativität ${ }^{9}$ im Bereich sexueller Devianz und die damit einhergehenden Konflikte bedeuteten nicht nur Überschneidungen und Kollisionen auf der normativen Ebene, sondern damit verknüpft waren auch Probleme der Zuständigkeiten und Strafkompetenzen. Wie die neuere Forschung gezeigt hat, konnten unterschiedliche Sexualdelikte von Kriminalgerichten, der geistlichen Gerichtsbarkeit, lokalen, genossenschaftlichen Frevel- und Rügegerichten oder der niederen Amtsund Policeygerichtsbarkeit verfolgt und geahndet werden, die über unterschiedliche Sanktionskompetenzen verfügten. ${ }^{10}$ Im frühneuzeitlichen Alten Reich hatten sich keine einheitlichen trennscharfen rechtlichen Kriterien herausgebildet, welche Institutionen welche Formen von sexueller Devianz verfolgten und straften. Als grobe Abgrenzung diente wie auch allgemein die Unterscheidung zwischen peinlichen Male-

\footnotetext{
${ }^{7}$ ELLRICHSHAUSEN, Die uneheliche Mutterschaft; GÜNTHER, Sittlichkeitsdelikte 121-148; DIES., Die Behandlung der Sittlichkeitsdelikte.

${ }^{8}$ Vgl. als Überblick: KOCH, Maior dignitas est; HULL, Sexualstrafrecht 221-234; HEHENBERGER, Sexualstrafrecht 101-118.

${ }^{9}$ Dazu allgemein: DuvE, Multinormativität 88-101.

${ }^{10}$ BECKER, Leben und Lieben; BREIT, Leichtfertigkeit; GleiXner, Das Mensch; BurghartZ, Zeiten der Reinheit; KLAMMER, Unehren.
}

fizstrafen (Leibes- und Lebensstrafen) und bürgerlichen Strafen (Geldbußen, Turmhaft), die sich aber mit der Ausdifferenzierung der Sanktionen durch die Policeygesetzgebung kaum strikt anwenden ließ. Vielmehr überschnitten sich - wie auch in anderen Rechts- und Policeybereichen - Normen, Zuständigkeiten und Sanktionskompetenzen, wozu noch erhebliche regionale Variationen der jeweiligen Obrigkeiten, Jurisdiktionen, Territorien und Städte hinzukommen konnten. Dies eröffnete für betroffene Akteure bzw. die jeweils von Verfolgung und Strafe betroffenen Frauen und Männer auch Spielräume, um sexuelle Devianz im Kontext von, guter Policey‘ und Strafjustiz auszuhandeln, wie im Folgenden ebenfalls gezeigt werden soll.

Entsprechend vielfältig gestaltet sich daher auch die neuere Forschung zu sexueller Devianz im frühneuzeitlichen Alten Reich, insbesondere im Hinblick auf die Praxis der Strafjustiz und die Erforschung von Kriminalität, die meist aus geschlechtsspezifischer Perspektive analysiert werden. ${ }^{11}$ Im Folgenden kann daher nur für die normative Ebene anhand der im Repertorium der Policeyordnungen der Frühen Neuzeit erfassten Normen ein genereller Überblick gegeben werden, ${ }^{12}$ der für die konkrete Umsetzung und Strafverfolgung am Beispiel des von mir untersuchten Kurfürstentums Mainz exemplarisch konkretisiert wird. ${ }^{13}$ Ausgehend von der Entwicklung der Policeygesetzgebung im Alten Reich zwischen 1500 und 1800 lassen sich

\footnotetext{
11 Überblick: SCHWERHOFF, Kriminalitätsforschung 151-164; weiterhin: UlBRICHT, Von Huren und Rabenmüttern; RUBLACK, Magd, Metz' oder Mörderin.

12 HÄRTER, STOlleis, Repertorium, das die Policeygesetze von 21 Ländern/Territorien (mit „Nebenterritorien") und 8 (Reichs-)Städten erschließt; diese werden im Folgenden mit Bandangabe, unter Angabe von Territorium/Stadt, laufender Nummer, Form und Datum zitiert.

${ }^{13}$ HÄRTER, Policey und Strafjustiz 820-929, auf der Basis der Kurmainzer Policey- und Kriminalakten.
} 
für den Bereich der Policeynormen zu devianter Sexualität und Ehe zwei Phasen des beschleunigten Wandels im 16. und im 18. Jahrhundert unterscheiden, die Grafik 1 (Seite 28) zeigt.

Nachdem bereits im 15. Jahrhundert vor allem größere Städte erste weltliche Ordnungsgesetze erlassen hatten, die auf Kriminalisierung und Disziplinierung des Sexualverhaltens zielten, lässt sich eine deutliche Intensivierung im Zeitalter von Reformation und Konfessionalisierung feststellen. Die Intensivierung der Kriminalisierung mittels Ordnungsgesetzgebung im 16. Jahrhundert war Teil der evangelischen wie der (später einsetzenden) katholischen Konfessionalisierung und der damit einhergehenden Sittenzucht und Ehegesetzgebung, die sich auf der normativen Ebene in Ehe- und Kirchenordnungen und zahlreichen Zucht- und Sittengesetzen manifestierte. ${ }^{14}$ Zwar lässt sich die katalytische Wirkung von protestantischer und katholischer Konfessionalisierung klar aufzeigen, ursächlich verantwortlich für die Regulierung und Kriminalisierung von Ehe und Sexualität war diese jedoch nicht, da sich entsprechende Normen bereits in der früheren Ordnungsgesetzgebung sowie dem Kirchen- und Strafrecht nachweisen lassen. ${ }^{15}$

So kriminalisierten mit meist arbiträren Strafandrohungen Ordnungsgesetze der Reichsstädte Frankfurt von 1425, 1451, 1452 und 1468 oder Köln von 1435, 1437 und 1450 Prostitution/Kuppelei sowie Unzucht und Ehebruch; Kurpfälzer Policeyordnungen und Mandate von 1465 und 1493 Unzucht, Bigamie, Konkubinat und Ehebruch; eine Magdeburger Ordnung von 1484 Unzucht und Konkubinat; die brandenburgischen Stadtordnungen von 1502 und 1515 Unzucht und öffentlichen Ehebruch, ebenso wie eine Kurmainzer Ordnung für die Stadt Walldürn von 1492 und die bayerische Landesordnung von

\footnotetext{
${ }^{14}$ SIBETH, Eherecht; WeStPHAL, Kirchenzucht; FRASSEK, Eherecht; VOLTMER, Unzucht.

${ }^{15}$ Vgl. nur SCHWAB, Grundlagen.
}

1504. ${ }^{16}$ Zudem lassen sich die Ehe- und Kirchenordnungen nicht ausschließlich dem Bereich des kirchlichen Rechts zuordnen, sondern sie weisen zahlreiche Überschneidungen mit der allgemeinen Policeygesetzgebung auf und nahmen vielfach die Gestalt von ,Kirchenpoliceyordnungen' an, die allgemein "Sittlichkeit" und insbesondere sexuelle Devianz regulierten und auch kriminalisierten. ${ }^{17}$

Die Amalgamierung von Kirchen- und Sittenzucht und "guter Policey" zwecks Regulierung und Kriminalisierung sexueller Devianz wird in einigen Ordnungen auch ausdrücklich benannt: „Des Radts der Alten Stadt Magdeburgk Ordnung vbern Ehebruch/ Gelübden/ Wirdschafften vnd Kleidung" aus dem Jahr 1544 erging nicht nur zu „Gödtlichen Lobe/ Glori/ vnd Ehr", sondern wollte „vberflussigkeit und vnordenung" bekämpfen, „zu erhaltung vnserer Stadt Policey/ vnd bürgerlicher narunge, damit jeder vor Gott vnd der welt gebessert werde zum gutt Christlich Exempel“. Der Disziplinierungsanspruch der Ordnung richtete sich insbesondere gegen verschiedene Formen sexueller Devianz, darunter Ehebruch, Unzucht, außerehelicher Geschlechtsverkehr und Schwängerung, Kuppelei, Hurerei, Bigamie und Notzucht, die detailliert kriminalisiert und mit unterschiedlichen Geld-, Haft-,

\footnotetext{
${ }^{16}$ HÄRTER, STOLLEIS, Repertorium 5: Frankfurt 340 Ordnung 1425, Frankfurt 493 Verordnung 1451, Frankfurt 500 Verordnung 29. 10. 1452, Frankfurt 1563 Policeyordnung 20. 3. 1468; HÄRTER, STOLLEIS, Repertorium 6: Köln 443 Morgensprache 1435, Köln 454 Statuten 15.6.1437, Köln 595 Morgensprache 1450, HÄRTER, STOLLEIS, Repertorium 3: Kurpfalz 18 Ordnung 17.7.1465, Kurpfalz 19 Ordnung 19.8.1465, Kurpfalz 20 Mandat 20.12. 1465, Kurpfalz 48 Stadtordnung 9.1493; HÄRTER, STOLLEIS, Repertorium 2: Magdeburg 6 Ordnung 24.8. 1484, BrandenburgPreußen 9 Ordnung 14.10.1502, BrandenburgPreußen 16 Ordnung 11.7.1515; HÄRTER, STOLLEIS, Repertorium 1: Kurmainz 25 Stadtordnung 27. 7. 1492; HÄRTER, STOLLEIS, Repertorium 3: Bayern 54 Landesordnung 15. 8. 1501.

${ }^{17}$ HÄRTER, Kirchenzucht 31-48; vgl. als Beispiel DOBRAS, Ratsregiment.
} 
Verweisungs-, Leibes- und Todestrafen bedroht wurden, wobei Männer und Frauen (bei gleichem Tatbestand) ausdrücklich gleichartig bestraft werden sollten. ${ }^{18}$ Deutlich verweist die Ordnung auf die allgemeine Leitkategorie der "guten Policey“, welche die Beseitigung von Unordnung und die Erhaltung der "Nahrung" einschließt. ${ }^{19}$

Die im 16. Jahrhundert zunehmende Regulierung und Kriminalisierung sexueller Devianz in der Policeygesetzgebung steht folglich in einem weiteren Kontext einer allgemeinen Ordnungskrise, die auch als „Verfall der Sitten“ und Strafe Gottes für abweichendes Verhalten, gerade im Bereich von Sittlichkeit und Sexualität wahrgenommen wurde. Die Obrigkeiten reagierten darauf mit Policeyordnungen, die in diesem Zusammenhang auch Gotteslästerung und Fluchen, die Kleiderordnung, übermäßigen Aufwand, Glücksspiel, die Festkultur (Zutrinken/Trinken, Tanzen, Hochzeit) sowie Eheschließung/Eheführung erfassten. Beispielsweise regulierten und kriminalisierten die Ulmer Zuchtordnungen von 1558, 1574 und 1582 neben Unzucht, Ehebruch, Prostitution und Kuppelei auch die Gotteslästerung, Aufwand/Luxus der Kleidung, Eheschließung/ Ehescheidung, Glücksspiel, Zutrinken und Trunksucht und den Wucher. Ähnliche Kombinationen finden sich in der Ordnungsgesetzgebung zahlreicher Obrigkeiten. ${ }^{20}$ Bezüglich sexueller Devianz zielten diese Ordnungen auch auf Prävention, z.B. durch das Verbot unzüchtiger Kontakte in Spinnstuben, von „üppigen“ (sexuell

\footnotetext{
${ }^{18}$ Des Radts der Alten Stadt Magdeburgk Ordnung vbern Ehebruch/ Gelübden/ Wirdschafften vnd Kleidung (Magdeburg 1544).

${ }^{19}$ Vgl. dazu SimOn, Gute Policey; HärTER, Policey.

${ }^{20}$ HÄRTER, STOLLEIS, Repertorium 8: Ulm 2194 Zuchtordnung 7.12.1558, Ulm 2499 Zuchtordnung 24. 11. 1574, Ulm 2600 Zuchtordnung 12. 1. 1581; vgl. dazu insgesamt HäRTER, STOLLEIS, Repertorium, insbesondere die im systematischen Index unter „1.4 Aufwand und Luxus", "1.5 Sittlichkeit; Ehe- und Familie; Sexualität“ und „2.1 Vergnügungen; Öffentliche Leichtfertigkeit" nachgewiesenen Policeygesetze.
}

aufreizenden) Tänzen und sonstigen „Leichtfertigkeiten“. ${ }^{21}$ Dass deviantes Sexualverhalten wie Ehebruch und außerehelicher Geschlechtsverkehr im 16. Jahrhundert tatsächlich signifikant zugenommen hätten, lässt sich allerdings nicht gesichert belegen. Eher bedingten das Bevölkerungswachstum, die Verknappung des Nahrungsspielraums und auftretende epidemische Seuchen ein wachsendes Konfliktpotential, das sich auch im Bereich von Ehe, Familie und Sexualität äußerte.

Insofern spielte der Kontext von Ehe und Familie ebenfalls eine Rolle für die intensivere Regulierung und Kriminalisierung devianter Sexualität. Unter der allgemeinen Leitvorstellung guter Ordnung und Policey avancierte die Ehe in der obrigkeitlichen Ordnungsgesetzgebung zu einem zentralen, schützenswerten Rechtsgut. Allein in ihren legalen Formen sollte noch eine ausschließlich der Fortpflanzung dienende Sexualität stattfinden. Zahlreiche Ordnungsgesetze reglementierten Ehe und Familie und kriminalisierten gleichzeitig deviantes Sexualverhalten, was zu einer weiteren Ausdifferenzierung der strafbaren Handlungen und Delikte führte, z.B. bezüglich der "Winkelehen" oder "heimlichen Ehen" und der Einhaltung der Bedingungen der Eheschließung (Elternkonsens, Inferenda, Trauung durch Priester, verbotene Verwandtschaftsgrade).

Neben Religion/Konfessionalisierung und Ehe/ Familie entwickelte sich die Kriminalisierung sexueller Devianz in einer intensiven Wechselwirkung mit dem Strafrecht, die sich bereits in der ersten Hälfte des 16. Jahrhunderts auf der reichsrechtlichen Ebene zeigte. Die Peinliche Halsgerichtsordnung Karls V. von 1532 kriminalisierte Sodomie unter Gleichgeschlechtlichen oder mit Tieren sowie Notzucht/Vergewaltigung und drohte die Todesstrafe an. Hinzu kamen Inzest, Jungfrauenschwächung und Entführung

\footnotetext{
${ }^{21}$ HÄRTER, Fastnachtslustbarkeiten; JUNG, Körperlust und Disziplin.; KelLER, Feierfreuden.
} 
sowie Ehebruch, Bigamie, Prostitution und Kuppelei, die peinlich, nach den kaiserlichen Rechten, nach Rat der Rechtsverständigen und Gestalt der Personen und Umstände mit Todesstrafe, Landesverweisung, Pranger, Prügel „oder anderm“ gestraft werden konnten. Die Strafandrohung blieb folglich arbiträr und konnte durch die Normgebung der Reichsstände und Obrigkeiten und auch in der Justizpraxis den Umständen angepasst werden.22 Gleichzeitig kriminalisierten die Reichspoliceyordnungen von 1530 und 1548 sexuelle Aktivitäten außerhalb der Ehe: „offentliche Ehebruch / unnd andere leichtfertige unnd unzimliche beiwonungen" (gemeint war vor allem das Unzuchtsdelikt) wurden verboten und sollten „von der Oberkeyt ernstlich an Leib oder gut / nach gestalt unnd gelegeneyt der personen / unnd der verwürckung gestrafft werden"; dies galt auch für das Konkubinat und die Kuppelei. ${ }^{23}$ Im Vergleich zur Carolina war die Strafandrohung noch unbestimmter, ermöglichte aber ebenfalls, dass die Obrigkeiten sexuelle Devianz mittels eigener Ordnungs- und Strafgesetzgebung weiter kriminalisieren und ex officio verfolgen konnten. Wesentlich war dabei die Festschreibung der „Öffentlichkeit" als Tatbestandsmerkmal, denn dadurch konnte die Obrigkeit bei öffentlich wahrgenommener sexueller Devianz ein Strafverfahren unabhängig von einer Klage in Gang bringen, z.B. auf der Basis von Gerede, Gerüchten oder einer Anzeige. Die Policeygesetze unterstützten folglich, dass praktisch alle Sexualdelikte im Rahmen eines öffentlich-inquisitorischen Straf- oder Policeyverfahrens gemäß Offizial- und Instruktionsgrundsatz verfolgt und damit potentiell der Kriminaljustiz zugeordnet

\footnotetext{
22 Schroeder, Peinliche Gerichtsordnung, Art. 116123; vgl. allgemein zur Bedeutung der Carolina und zum Forschungsstand: HÄrTER, Strafrechts- und Kriminalitätsgeschichte.

${ }^{23}$ WeBER, Reichspolizeiordnungen, hier Art. 33 (1530) 160f. und der zitierte Art. 25 (1548) 201f.; vgl. HÄRTER, Policeygesetzgebung.
}

werden konnten. ${ }^{24}$ Die Carolina von 1532 und die Reichspoliceyordnungen von 1530 und 1548 zeigen folglich exemplarisch die Komplementarität von peinlichem Recht und Policeygesetzgebung und sie verdichteten die Kriminalisierung sexueller Devianz, was sich katalytisch auf die entsprechende Ordnungsgesetzgebung der Reichsstände auswirkte.

Zahlreiche Reichsstände orientierten sich an den Ordnungen von 1530/1548 und 1532 und integrierten die Verbote des öffentlichen Ehebruchs, der Unzucht, der Kuppelei und der Prostitution über Kirchen- und Eheordnungen hinaus auch in ihre Landes- und Policeyordnungen, so die Zweibrücker Landesordnung von 1534, die kurpfälzische Policeyordnung von 1546, die bayerischen Landes- und Policeyordnungen von 1553, 1578, 1598 und 1616, die jülich-bergische Policeyordnung von 1558, die württembergischen Landesordnungen von 1536 und 1552, 1567 und zahlreiche weitere. ${ }^{25}$ Auffällig ist, dass es sich nicht nur um evangelische Landesherrschaften handelte, sondern dass auch die katholischen und geistlichen Reichsstände, letztere allerdings erst später und meist nach dem Trienter Dekret „Tametsi" von 1563, gleichartige Verbote zu Unzucht, öffentlichem Ehebruch, Konkubinat und z.T. auch Inzest in ihre Ordnungen aufnahmen, so Kurmainz 1582, Kurtrier in die Kirchen- und Policeyordnung von 1589 oder Kurköln in die Policeyordnung von 1595. ${ }^{26}$

${ }^{24} \mathrm{Zu}$ den Verfahren: HÄRTER, Strafverfahren.

${ }^{25}$ HÄRTER, STOlleis, Repertorium 3: PfalzZweibrücken 32 Landesordnung 24. 2. 1534, Kurpfalz 170 Policeyordnung 17.6. 1546, Bayern 307 Landesordnung 09.1.1553, Bayern 432 Landesordnung 12. 6. 1578, Bayern 532 Landgebot 13.3.1598, Bayern 650 Landes- und Policeyordnung 28. 9. 1616; JülichBerg 53, Policeyordnung, 15. 5. 1558; HÄRTER, STOLLEIS, Repertorium 4: Württemberg 73 Landesordnung 1.6.1536, Württemberg 127 Landesordnung 2. 1. 1552; Württemberg 195 Landesordnung 17. 8. 1567.

${ }^{26}$ HÄRTER, STOLLEIS, Repertorium 1: Kurmainz 133 Ordnung/Edikt 22. 1. 1582, Kurtrier 108 Kirchen- \& 
Darüber hinaus zeigte sich seit dem letzten Drittel des 16. Jahrhunderts vor allem in evangelischen Landesherrschaften eine Tendenz zu einer stärker strafrechtlichen Ausrichtung der Ordnungsgesetzgebung, einhergehend mit einer Ausdifferenzierung von Tatbeständen, Verfahren und Sanktionen, die sich in spezifischen Strafmandaten und Strafordnungen manifestierte. „Pfaltzgraff Ludwigs, Churfürstens, Mandat vnd Constitution, welcher Gestalt die Blutschanden, Notzucht, Raub oder Entführung, auch Ehebruch, Hurerey vnnd dergleichen Laster zu straffen," behandelte 1583 nicht nur eine breite Palette von Sexualdelikten, die von sexueller Gewalt (Vergewaltigung, Entführung) über Ehebruch und Inzest bis $\mathrm{zu}$ unbestimmten Unzuchtsdelikten reichte, sondern drohte für Ehebruch männlichen und weiblichen, verheirateten und ledigen Delinquenten Todesstrafen an: die Gerichte sollten, „wann ein Ehe/ oder ledige MannsPerson/ bey einer Ehefrawen ergriffen/ vnd deß Ehebruchs uberzeuget/ beyde Ehebrecher vnnd Ehebrecherin mit dem Schwert/ oder Wasser vom Leben zum Todt strafen". ${ }^{27}$ Auch das Herzogtum Württemberg erließ 1586 ein umfangreiches Mandat, in dem Unzucht/Hurerei, Ehebruch, Bigamie, Inzest, Vergewaltigung und verschiedene Sodomie-Verbrechen (Homosexualität, Bestialität und Nekrophilie) mittels einer „erhöhung vnd schärpffung deren biß daher gebrauchten [ge]ringen und milten Straff" kriminalisiert wurden, weil diese "grewlich hochsträfflich Laster [...] allzvvil vberhand genommen" hätten. Für die Strafverfolgung von "Ampts wegen" war ausdrücklich die Lokalverwaltung auch ohne vorherige rechtliche Klage zuständig. Delikte und Strafandrohungen waren für Frauen und Männer

Policeyordnung 16.8.1589, Kurköln 16 Policeyordnung 4. 11. 1595.

${ }^{27}$ HÄRTER, STOLLEIS, Repertorium 3: Kurpfalz 366 Mandat 1583, online:

http://nbn-resolving.de/urn:nbn:de:bvb:12-

bsb00039842-5 [Zugriff 10. 7 .2018]. nicht nur gleich (soweit dies möglich war), sondern das Mandat fokussierte ausdrücklich auf Männer bzw. männliche Kriminalität, wie insbesondere ,Verführung' und ,Schwächung' unbescholtener ,Jungfrauen', sexuelle Gewalt und Sodomie-Verbrechen. Die angedrohten Strafen reichten von Geldbußen und mehrwöchiger Haft über Schand- und Ehrenstrafen, Kirchenbuße, Prügel und Landesverweisung bis $\mathrm{zu}$ Todesstrafen für Inzest ersten Grades; der Ehebruch sollte im ersten Fall nur mit Haft, im Wiederholungsfall aber „vermög der peinlichen Halßgerichtsordnung" ebenfalls mit Todesstrafe geahndet werden. Hinsichtlich des Verfahrens und der Strafen räumte das Mandat den zuständigen Amtsträgern und Gerichten einen relativ weiten Spielraum ein, sexuelle Devianz nach Gelegenheit der Person, Umständen, Verhandlung und richterlichem Ermessen zu ahnden. ${ }^{28}$ Auch die "StraffOrdnung wie künfftig im Hertzogthum Zweybrücken die Hurerey und Unzucht an ledigen und ehelichen Personen zu bestraffen" kriminalisierte 1605 (und in nachfolgenden Ausgaben bis 1723) detailliert Unzucht/Hurerei, Ehebruch, Prostitution/Kuppelei, Inzest, Sodomie und Vergewaltigung. Sie stellte zudem deutlich den $\mathrm{Zu}-$ sammenhang zwischen außerehelicher Schwangerschaft bzw. nichtehelichen Kindern und sexueller Devianz in Form von Unzucht und Ehebruch her, verschärfte die Strafen (ebenfalls Todesstrafe bei mehrfachem Ehebruch), statuierte ein inquisitorisches Policeystraf- und Kriminalverfahren und die obrigkeitliche Verpflichtung der Verfolgung sexueller Devianz im Rahmen von Policey und Strafjustiz. ${ }^{29}$ Diese Entwicklung zeigt sich auch in der Ordnungsgesetzgebung der Reichsstädte, wie z.B. in der 1594 erlassenen

\footnotetext{
${ }^{28}$ HÄRTER, STOLLEIS, Repertorium 4: Württemberg 240 Verordnung 21.5.1586, hier zitiert nach REYSCHER, Vollständige Sammlung 4, 443-450.

${ }^{29}$ HÄRTER, STOLLEIS, Repertorium 3: Pfalz-Zweibrücken 137 Strafordnung 13. 5. 1605, letzte Ausgabe vom 23. 7. 1723 (gedruckt Zweibrücken 1724).
} 
"Constitution und Satzung eines Ersamen Rahts der Freyen Reichsstatt Strassburg, wie der Ehebruch und andere Unzucht hinfüro gestrafft werden sollen", sowie in verschiedenen Ordnungen und Mandaten der Reichstädte Frankfurt am Main, Köln und Ulm. ${ }^{30}$ Auch katholische bzw. geistliche Reichsstände wie Bayern und Kurmainz erließen - allerdings in etwas geringerem Umfang und insgesamt zeitlich später - umfassende Einzelgesetze, die sich ausschließlich mit den genannten Sexualdelikten bzw. sexueller Devianz beschäftigten und durch eine Verschärfung der Strafandrohung und eine weitgehende Zuständigkeit und Pflicht $\mathrm{zu}$ obrigkeitlicher Strafverfolgung gekennzeichnet waren. ${ }^{31}$

Hinsichtlich der quantitativen Verteilung der Sexualdelikte in der Policeygesetzgebung von 19 Territorien und acht Reichsstädten, die Grafik 2 (Seite 29) zeigt, ergibt sich über den gesamten Untersuchungszeitraum (1500 bis 1799) eine klare Dominanz der Unzuchtsdelikte mit 45 bis 55 Prozent, gefolgt vom Ehebruch mit etwas über 20 Prozent und Prostitution/Kuppelei mit 10 bis 15 Prozent: letztere wurde in den Städten intensiver normiert. Bei den Delikten, die eng mit der Eheschließung verbunden sind, spielen Bigamie und Konkubinat kaum eine Rolle und lediglich der Inzest (meist Regulierung der Verwandtschaftsgrade, bei denen eine Eheschließung verboten

\footnotetext{
${ }^{30}$ Constitution und Satzung eines Ersamen Rahts der Freyen Reichsstatt Strassburg, wie der Ehebruch und andere Unzucht hinfüro gestrafft werden sollen (Straßburg 1594); HäRTER, STOLLEIS, Repertorium 5: Frankfurt 1408 Verordnung 24. 5. 1579, Frankfurt 1638 Verordnung 10. 10.1598, Frankfurt 1943 Verordnung 14. 11. 1620; HÄRTER, STOLLEIS, Repertorium 6: Köln 4747 Ratsbeschluß 17.12.1631; HÄRTER, STOLLEIS, Repertorium 8: Ulm 2194 Zuchtordnung 7. 12. 1558, Ulm 2499 Zuchtordnung 24. 11. 1574, Ulm 2600 Zuchtordnung 12.1.1581, Ulm 2930 Zuchtordnung 15. 10. 1605.

${ }^{31}$ HÄRTER, STOLLEIS, Repertorium 3: Bayern 514 Mandat 15. 1. 1595, Bayern 521 Mandat 25. 4. 1596, Bayern 804 Mandat 20. 9. 1635; HÄrTER, STOLLEIS, Repertorium 1: Kurmainz 209, Mandat, 20. 9. 1658.
}

war) wurde intensiver normiert. ${ }^{32}$ Dagegen fand das schwerste Sexualverbrechen "Sodomie" nur geringe Aufmerksamkeit in der Policeygesetzgebung, da diesbezüglich das gemeine Recht bzw. Strafrecht offenbar ausreichte und sich kein Regulierungsbedarf im Hinblick auf die gute Ordnung ergab (oder diese Form sexueller Devianz in der Gesetzgebung möglichst verschwiegen werden sollte). ${ }^{33}$

Die Begründungen der Einzelgesetze rekurrierten wie die Landes- und Policeyordnungen auf die christliche Ordnung und Gottes Strafe, die Abschaffung von Sünde, Schande und Laster sowie die Herstellung von bürgerlicher Zucht und guter Ordnung, wobei neben Kirchenrecht bzw. Kirchen- und Eheordnungen öfters auch auf das gemeine bzw. das Reichsrecht verwiesen wurde. $\mathrm{Ob}$ um die Wende vom 16. zum 17. Jahrhundert deviantes Sexualverhalten bzw. die entsprechenden Sexualdelikte tatsächlich signifikant zugenommen hatten, wie die meisten Ordnungsgesetze behaupteten, lässt sich kaum empirisch belegen. Eher scheint es, dass die unterschiedlichen Obrigkeiten die Möglichkeit nutzten, um im Kontext der guten Policey die strafrechtlichen Normen auszuweiten, Verfahren und Strafkompetenzen (auch im Hinblick auf intermediäre Gewalten) genauer festzuschreiben und damit insgesamt sexuelle Devianz primär der obrigkeitlichen Strafjustiz zuzuordnen, um so auch eine stärkere Abschreckung und Disziplinierungswirkung zu erzielen. Dies erfolgte jedoch keineswegs als stringente Verstaatlichung, sondern lokale Organe, niedere oder kirchliche Gerichte blieben weiterhin für die Durchführung von Verfahren und die Bestrafung von leichteren Unzuchtsdelikten zuständig, soweit keine peinlichen, sondern typische niedergerichtlich-policeyliche Strafen (Geldbußen, kurze Haft) verhängt wurden. Schwerere Delikte wie Ehebruch, Inzest oder

\footnotetext{
${ }^{32} \mathrm{Vgl}$. dazu auch JARZEBOWSKI, Inzest.

${ }^{33}$ Dazu allgemein: Susanne HeHENBerger, Unkeusch wider die Natur.
} 
Sodomie blieben der peinlichen Strafgerichtsbarkeit vorbehalten, die aber auch leichtere Vergehen wie Fornikation und Prostitution mittels arbiträrer, außerordentlicher Strafen verfolgen konnte. Insofern führte die zunehmende Reglementierung sexueller Devianz zu einer Ausweitung und Ausdifferenzierung der außerordentlichen, arbiträren Strafen im Bereich der Sexualdelikte und erlaubte eine Flexibilisierung der Sanktionsmöglichkeiten. Dies beeinflusste die Praxis der Strafjustiz im Hinblick auf eine Ausdifferenzierung und Abmilderung der Strafen, da gute Policey - Normen wie Ziele/Zwecke - ein Abweichen von der peinlichen "poena ordinaria“ und die Anwendung von außerordentlichen, arbiträren Strafen ermöglichte und legitimierte.

Die Kurmainzer Strafpraxis der Landesregierung (als einzigem höchstem Strafgericht) lässt entsprechende Wechselwirkung zwischen Policeygesetzgebung und Strafjustiz erkennen. ${ }^{34}$ Die Normgebung setzte, wie allgemein, im 16. Jahrhundert nach den Beschlüssen des Konzils von Trient ein und intensivierte sich bis zum Beginn des Dreißigjährigen Krieges im Kontext einer verstärkten Disziplinierung und Konfessionalisierung der Untertanen. Erst gegen Ende des 17. und dann vor allem um die Mitte des 18. Jahrhunderts kam es zu einer weiteren Intensivierung der Ordnungsgesetzgebung des Kurstaates im Bereich sexueller Devianz sowie von Ehe und Familie. Hintergrund der normativen Entwicklungen war weniger eine tatsächliche allgemeine Zunahme sexueller Devianz, sondern öfters führten auch einzelne Fälle, die öffentliches Aufsehen erregten, spezifische Problemkonstellationen wie Kompetenz- und Jurisdiktionskonflikte zwischen weltlichen und geistlichen Institutionen (Regierung und Generalvikariat) sowie Justiznutzungspraktiken der Untertanen zu einem Anstieg der Normgebung und der Strafverfolgung. Letztere

\footnotetext{
${ }^{34}$ Das Folgende, soweit nicht anders vermerkt, nach: HäRTER, Policey und Strafjustiz 533-557 und 820-929.
}

lässt deutliche Einflüsse der Policeygesetzgebung erkennen, ohne dass dies als eine rigorose oder lineare Durchsetzung der Normen interpretiert werden könnte. Die Kriminalisierung sexueller Devianz bedeutete zunächst, dass die Strafkompetenzen bis zum 18. Jahrhundert weitgehend zu den weltlichen Justiz- und Verwaltungsinstitutionen verlagert worden waren und vor allem die Landesregierung, die auch für die Policeygesetzgebung zuständig war, als Strafgericht alle Sexualdelikte von der einfachen Unzucht bis zur Sodomie verfolgte und meist strafte. Die geistlichen und lokalen Institutionen blieben freilich in das Untersuchungsverfahren und die Strafvollstreckung involviert. Diese Entwicklung führte bis zum 18. Jahrhundert zu einem starken Anstieg der im Rahmen der Strafjustiz verfolgten sexuellen Devianz, die rund 38 Prozent aller von der Landesregierung bearbeiteten Fälle (1566 mit 2951 beteiligten Delinquenten, davon 1557 Frauen und 1393 Männer) ausmachte (der Anteil der Delinquenten lag bei 39 und der der Delinquenz bei 37 Prozent). Der Verfolgungshöhepunkt lag zwischen 1740 und 1790 und im Hinblick auf die einzelnen Delikte ergibt sich eine quantitative Verteilung, die der Normgebung ähnelt, wie Tabelle 1 (Seite 29) zeigt.

Unzucht und Ehebruch dominierten eindeutig und nur der Inzest spielte noch eine quantitative Rolle, wobei es sich hier meist um Fälle entfernterer Verwandtschaftsbeziehungen handelt. Gleichzeitig zeigt sich auch eine Differenzierung und Milderung der Sanktionspraxis, in der keine signifikanten Unterschiede zwischen weiblichen und männlichen Delinquenten erkennbar sind. Bei den Strafen dominieren mit rund 65 Prozent Geldbußen und kurze Haftstrafen (Turm, Hausarrest für Frauen), die meist bei Unzuchtsdelikten verhängt wurden. Festungsbau, Zuchthaus und sonstige Arbeitsstrafen machen rund 15 Prozent aus und wurden bei den schweren Delikten (Sodomie, mehrfacher Ehebruch) verhängt; einfachen Ehebruch strafte die Landesregierung überwiegend mit der Kirchenbuße (ca. $5 \%$ ), die 
allerdings gegen Ende des 18. Jahrhunderts abgeschafft wurde; Landesverweise, Prügel, Schamund Ehrenstrafen (ca. 5 \%) kamen meist bei Prostitution/Hurerei zum Einsatz; Todesstrafen gab es keine, aber rund zehn Prozent der Kriminalverfahren wurden eingestellt. Diese differenzierte Strafpraxis entsprach durchaus den Vorgaben der Policeynormen, die meist arbiträre, außerordentliche Strafen nach "Gestalt der Umstände“35 androhten und damit den sanktionierenden Organen einen relativ großen Spielraum eröffneten, sexuelle Devianz nach unterschiedlichen Kriterien und Zwecken zu strafen.

Diese Flexibilisierung der obrigkeitlichen Verfolgung und Bestrafung sexueller Devianz im Kontext der guten Policey verstärkte Kompetenz- und Jurisdiktionskonflikte (in Kurmainz bezüglich der geistlichen Gerichtsbarkeit und der Sanktionskompetenzen lokaler Organe), erweiterte aber ebenfalls die Möglichkeiten der involvierten sozialen Akteure, Policeynormen und Strafjustiz zu nutzen: Insofern förderte die Policeygesetzgebung im Bereich sexueller Devianz Justiznutzung und das Aushandeln von Sanktionen. Zudem lag es im Interesse der guten Policey, dass sexuell deviante Männer gleichermaßen wie Frauen verfolgt, bestraft und diszipliniert werden sollten. Dementsprechend machte die Policeygesetzgebung in der Strafandrohung keine Unterschiede, sondern relativierte (oder überging) die im mittelalterlichen Strafrecht vorhandene Benachteiligung von Frauen (besonders bei Ehebruch und Unzucht). ${ }^{36}$ Auch dies konnte die Möglichkeiten sozialer Akteure - insbesondere von Frauen und ihren Familien - verstärken, auf der Basis von Policeynormen und mithilfe der obrigkeitlichen Strafverfolgung gegen sexuell deviante Männer (z.B. Ehemänner oder Väter außerehelich gezeugter Kinder) vorzugehen.

\footnotetext{
${ }^{35} \mathrm{Vgl}$. allgemein HolEnSTEIN, Die Umstände der Normen 1-46.

${ }^{36}$ Vgl. dazu auch Hull, Sexualstrafrecht 232; HEHENBERGER, Sexualstrafrecht 101-111.
}

Diese weitgehende "Gleichbehandlung" und die Intention der Disziplinierung sexuell devianter Männer ist in der Strafpraxis der Kurmainzer Strafjustiz deutlich erkennbar. Strafjustiz und gute Policey boten den betroffenen Akteuren/Delinquenten gerade im Bereich sexueller Devianz infrajustizielle Optionen: Supplikationen und die Gewährung von Gnade waren eine vielfach genutzte Option, um sexuelle Devianz und Strafen im Rahmen von guter Policey und Strafjustiz auszuhandeln. Seit dem spätem 17. Jahrhundert lässt sich in Kurmainz insgesamt eine Zunahme des Supplizierens und der Gnadengewährung bei Sexualdelikten feststellen, die meist zu einer Abmilderung von Strafen führten. Dies betraf z.B. den Ehebruch, wenn Ehepartner dem devianten Ehebrecher verziehen und dies mittels einer Supplikation, die oft policeylich-utilitaristische Argumente anführte, der zuständigen Landesregierung kommunizierten. Diese passte daraufhin Strafen an, wobei ebenfalls policeyliche Intentionen und Normen eine Rolle spielten. Zahlreiche Policeygesetze forderten Betroffene, Familienmitglieder und Hebammen zur Anzeige illegitimer Schwangerschaften/Geburten auf oder verordneten sogar eine Anzeigepflicht. Anzeigepflicht und geschlechtergleicher Disziplinierungsanspruch in Verbindung mit der Instruktions- und Wahrheitsmaxime des inquisitorischen Strafverfahrens ermöglichten aber auch Frauen, deviante Ehemänner anzuzeigen oder auf dem Supplikationsweg gegen Kindsväter und Dienstherren vorzugehen. So nutzen schwangere, unverheiratete Frauen im Kontext von Zivilklagen, die Eheversprechen oder den Unterhalt außerehelicher Kinder betrafen, Selbstanzeige und Supplikationen und zeigten das Unzuchtsdelikt sowie beide „Täter" an. Diese Form der Justiznutzung gründete auf dem Vorrang von Policey und Strafrecht vor dem Zivilrecht und den partiell übereinstimmenden Interessen von Frauen und Staat bezüglich der Verfolgung sexuell devianter Männer und der Sicherstellung von Unterhaltszahlungen (um z.B. die gemeindli- 
che/obrigkeitliche Fürsorge zu entlasten). ${ }^{37}$ Diese Formen der Nutzung von Policey und Strafjustiz im Bereich sexueller Devianz trugen folglich ebenfalls zur relativ hohen Verfolgungsintensität in Kurmainz im 18. Jahrhundert bei und sie lassen sich auch in unterschiedlichen Variationen für andere Obrigkeiten nachweisen. ${ }^{38}$

Justiznutzung und policeylich-utilitaristische Zwecke beeinflussten zunehmend die strafrechtliche Verfolgung sexueller Devianz und führten langfristig zu differenzierteren Sanktionsformen, milderen Strafen und einer Fiskalisierung des Strafens in der zweiten Hälfte des 18. Jahrhunderts. Dies manifestierte sich z.B. in der Abschaffung der öffentlichen, entehrenden Kirchenbußen sowie traditionaler Ausweisungs-, Ehrenund Schandstrafen, der Umwandlung schwerer Strafen in Geldbußen (auf dem Supplikationsund Gnadenweg) und den zunehmenden Dispensmöglichkeiten auch im Hinblick auf die Eheschließung bzw. das Inzestverbot. In der Strafpraxis und auf der normativen Ebene traten die religiös-sittlichen und auf Disziplinierung des Sexualverhaltens gerichteten Strafzwecke zurück und bevölkerungspoliceyliche, ökonomische, aufklärerische und letztlich auch fiskalischutilitaristische Zwecke verstärkten sich und prägten auch die Policeygesetzgebung (Grafik 3, Seite 30).

In der zweiten Hälfte des 18. Jahrhunderts lässt sich in den Territorialstaaten des Alten Reiches eine zeitlich etwas versetzte Intensivierung der Ordnungsgesetzgebung bezüglich der Unzuchtsdelikte und der Regelungsfelder Ehe/Familie feststellen, die von einer Entkriminalisierung und Abmilderung der Strafandrohungen ab den

\footnotetext{
${ }^{37}$ HëRTER, Kriminalität 101-134; DERS., Erfahrungen; DERS., Das Aushandeln von Sanktionen 243-274.

${ }^{38}$ GÜNTHER, Behandlung der Sittlichkeitsdelikte; HULL, Sexuality; ULBRICHT, Huren und Rabenmüttern; NUBOLA, WÜRGLER, Suppliche e gravamina; BECKER, Leben und Lieben; BREIT, Leichtfertigkeit; KLAMMER, Unzucht.
}

1750er Jahren begleitet war. Dies stand im Kontext einer sich wandelnden, stärker auf sozioökonomische Steuerung und neue gesellschaftspolitische Ordnungsziele ausgerichteten Policeygesetzgebung, insbesondere im Hinblick auf die territorialstaatliche "Peuplierungspolitik" und die "Bevölkerungspolicey“, die eine Bevölkerungszunahme und die Förderung von Eheschließungen zum Ziel hatte (und den Anstieg der Normierung in den 1750er und 1780er Jahren bewirkte). In diesem Kontext war auch die Kindsmorddebatte der Aufklärung angesiedelt, die die Entkriminalisierung von Unzucht und Ehebruch forderte ${ }^{39}$ und die sich ebenfalls in der Policeygesetzgebung niederschlug. Die Ordnungsgesetzgebung regulierte vor allem die Bedingungen und Voraussetzungen einer Eheschließung wie Vermögen und Nahrungsgrundlage, um die ökonomische Basis einer Ehe zu sichern und Verarmung und Sozialfürsorge zu vermeiden. Dazu gehörten weiterhin die Ableistung des Miliz- und Militärdienstes und das Ziel der Beschränkung der Reproduktionsmöglichkeiten von Unterschichten und Randgruppen. Die restriktive policeyliche Ehegesetzgebung verschärfte allerdings das Problem sexueller Devianz und wirkte sich daher ambivalent auf die Entwicklung der Unzucht bzw. deren Verfolgung aus, wie sich am Kurmainzer Beispiel zeigen lässt: Die Schließung oder Erhaltung einer Ehe konnte in der Justizpraxis zur Abmilderung der Strafen führen, die Verschärfung im Bereich der Ehegesetzgebung führte zu einem Anstieg vorehelicher Sexualaktivitäten bzw. deren Verfolgung, die aber von einer Entkriminalisierung der Unzuchtsdelikte begleitet war. Im Ergebnis führte dies in Kurmainz - und wohl auch insgesamt im Alten Reich - seit dem späten 18. Jahrhundert zu einem Anstieg der in der Frühen Neuzeit insgesamt eher niedrigen Illegitimitätsraten (die

\footnotetext{
${ }^{39} \mathrm{Vgl}$. aus der umfangreichen Literatur hier lediglich UlBRICHT, Kindsmord.
} 
selbstverständlich noch weitere sozioökonomische Gründe hatte). ${ }^{40}$

Das sich in der zweiten Hälfte des 18. Jahrhunderts zuspitzende ambivalente Verhältnis von Policey und Strafjustiz, die damit einhergehenden Jurisdiktions- und Zielkonflikte und administrativen Probleme, die flexible und oft mildere Strafpraxis und die Praktiken der Justiznutzung beeinflussten die Entkriminalisierung sexueller Devianz insbesondere im Hinblick auf die ,Massendelikte' Unzucht und Ehebruch, die folglich nicht nur Ergebnis des Aufklärungsdiskurses und einer steuernden Politik der Obrigkeiten war. ${ }^{41}$ Entkriminalisierung und Strafmilderung waren allerdings begleitet von einem Ausbau des Instrumentariums formeller Sozialkontrolle im Bereich der Gesundheits- und Medicinalpolicey (bezüglich der Hebammen und der Geburtshilfe) und auf der Ebene der lokalen Verwaltungen in Form von Unzuchtstabellen, Belohnungen, strafbewehrter Anzeigepflicht von Hebammen und Eltern, Überwachungs- und Meldepflichten von lokalen Amtsträgern und Geistlichkeit, Ehekonzessionen der weltlichen Behörden und Geburtshilfeeinrichtungen bzw. Gebäranstalten.

Die Wirkung von Policeynormen und staatlicher Verfolgung war und bleibt daher ambivalent: Die seit dem 16. Jahrhundert zunächst sinkende Illegitimitätsrate kann nicht unilinear und monokausal auf die repressive Sexualkontrolle des Staates und der Kirchen zurückgeführt werden. Insbesondere im lokalen Bereich eröffnete sich ein Handlungsspielraum, der von der Bevölkerung flexibel genutzt wurde, um selektiv mit den Kon-

\footnotetext{
${ }^{40}$ HÄRTER, Policey und Strafjustiz 924-929; HARMSZIEGLER, Illegitimität und Ehe 314ff.; BREIT, Leichtfertigkeit 267-294; KLAMMER, Unzucht 211-223; MAISCH, Unzucht 279-306; BECKER, Illegitimität und Herrschaft 157-179; und allgemein: PFISTER, Bevölkerungsgeschichte und historische Demographie, 1500-1800 (München 1994) 86-89.

${ }^{41}$ So Hull, Sexuality; TAEGER, Moralstrafrecht und das Ergebnis meiner Studie HÄRTER, Policey und Strafjustiz.
}

flikten im Bereich von Ehe und Sexualität umzugehen, d.h. nur bestimmte Delikte, Personen oder Gruppen wurden angezeigt. Andererseits perfektionierte der frühmoderne Territorialstaat im Rahmen der guten Policey Normen, Durchsetzungsinstrumentarium und Sanktionierung und verstärkte so den Zugriff auf das als abweichend definierte Sexualverhalten, das durchaus verfolgt und differenziert bestraft wurde. Dabei kam der Amalgamierung von Policey und Strafjustiz eine wichtige Funktion zu, die zwar weder eine vollständige repressive Normdurchsetzung noch sexuelle Disziplinierung bewirkte, aber durchaus eine steuernde Wirkung entfaltete. Denn die Policeynormen stimmten vielfach mit religiösen und sozialen Normen überein, ermöglichten eine Flexibilisierung des Strafrechts, weiteten die Optionen der Untertanen zur infrajustiziellen und selektiven Justiznutzung aus und erweiterten die obrigkeitlichen Spielräume zur Verfolgung und Sanktionierung sexueller Devianz. Von Kriminalisierung und Disziplinierung im Kontext der guten Policey konnten insbesondere Frauen profitieren, während die in der zweiten Hälfte des 18. Jahrhunderts einsetzende Entkriminalisierungstendenz eher die Männer begünstigte. Allerdings bewirkte die Policeygesetzgebung in der Frühen Neuzeit keine eindeutige lineare Kriminalisierung oder Entkriminalisierung devianter Sexualität, sondern war durch widersprüchliche, teils kollidierende Ziele und Tendenzen gekennzeichnet, die an der Wende zum 19. Jahrhundert zu der eingangs exemplarisch skizzierten Problemlage führten: Sexuelle Devianz blieb weiterhin ein Bereich, in dem Ordnungsnormen, Strafrecht, Justiz, Verwaltung, Kirchen und Gesellschaft die „unwiderstehliche Allmacht des Geschlechtstriebes" mit sehr unterschiedlichen, von Kriminalisierung und Disziplinierung bis zur präventiven Regulierung und Liberalisierung reichenden Konzepten einhegen wollten. 
Grafik 1: Policeynormen Sexualdelikte \& Ehe 19 Territorien und 8 Reichsstädte 1500-1799

(Gezählt sind auf der Basis von HäRTER, STOLleIs, Repertorium, nicht Gesetze, sondern einzelne Policeymaterien nach dem dreistufigen Materienindex des Repertoriums: 5 Bereiche, 25 Gruppen und ca. 200 Policeymaterien).

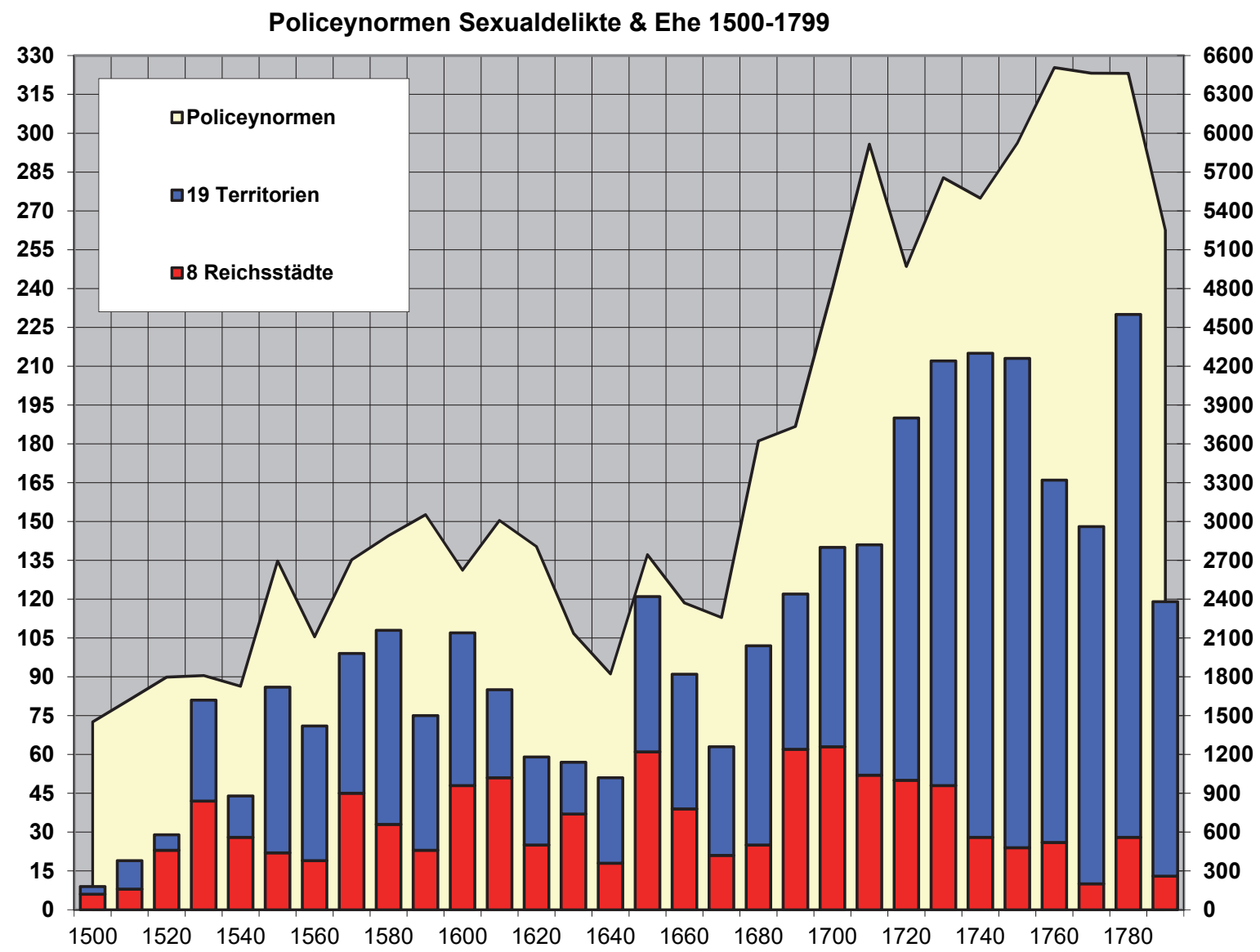


Grafik 2: Policeynormen Sexualdelikte 19 Territorien und 8 Reichsstädte 1500-1799

(Datengrundlage HÄRTER, STOLLEIS, Repertorium)

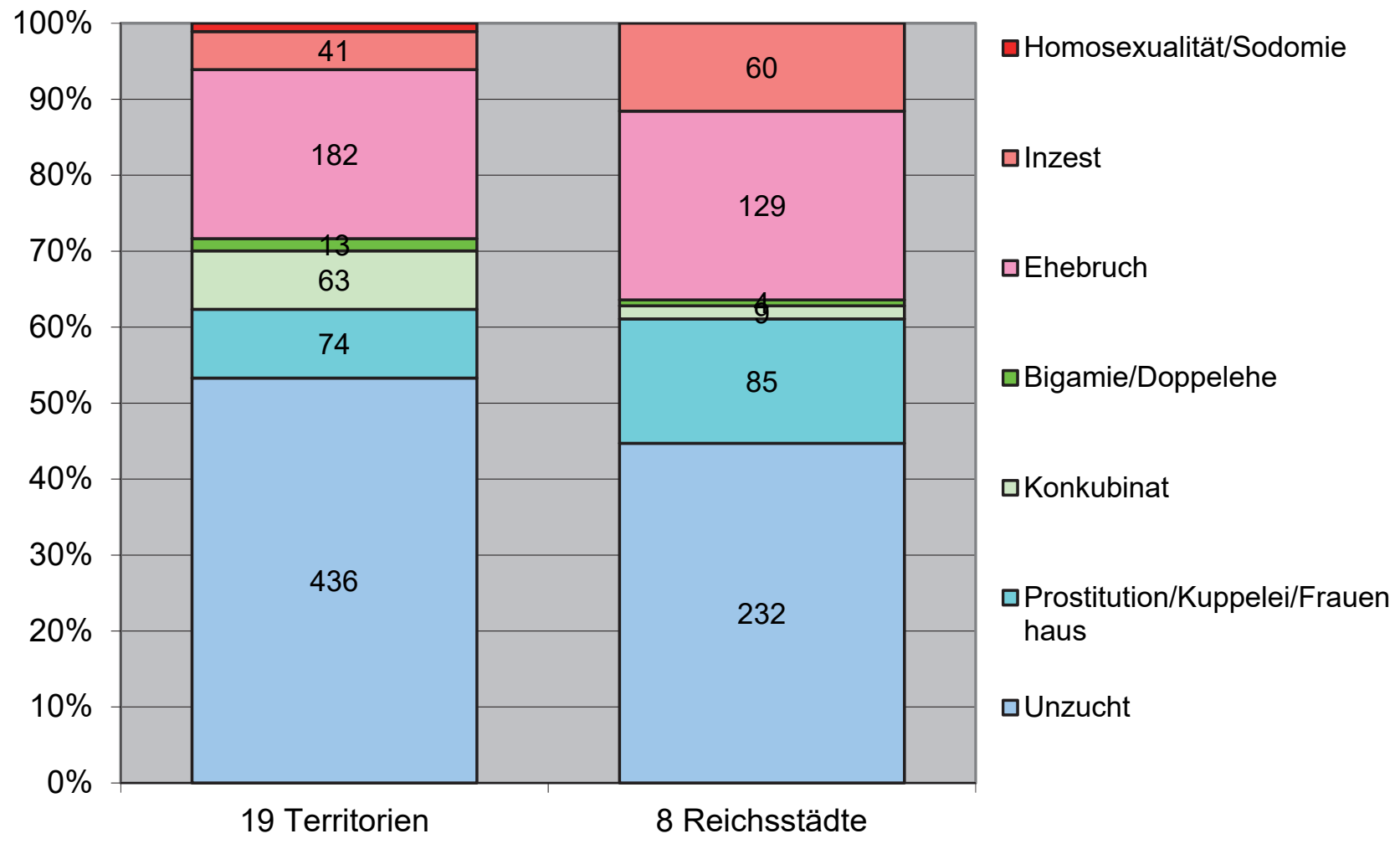

Tabelle 1: Häufigkeitsverteilung Sexualdelikte höchste Strafgerichtsbarkeit Kurmainz 1560-1802

\begin{tabular}{|l|r|r|}
\hline Sexualdelikte & \multicolumn{1}{|c|}{ Delinquenz } & \multicolumn{1}{c|}{ Fälle } \\
\hline Unzucht & $1806=67,7 \%$ & $948=69 \%$ \\
\hline Ehebruch & $576=21,6 \%$ & $277=20,2 \%$ \\
\hline Konkubinat & $21=0,8 \%$ & $10=0,7 \%$ \\
\hline Bigamie / Eheschließung & $29=1,1 \%$ & $19=1,4 \%$ \\
\hline Inzest & $168=6,3 \%$ & $85=6,2 \%$ \\
\hline Entführung & $2=0,1 \%$ & $1=0,1 \%$ \\
\hline Prostitution \& Kuppelei & $60=2,2 \%$ & $28=2,0 \%$ \\
\hline Exhibitionismus & $1=0 \%$ & $1=0,1 \%$ \\
\hline Sodomie & $4=1,5 \%$ & $4=0,3 \%$ \\
\hline Gesamt & $\mathbf{2 6 6 7}=\mathbf{1 0 1} \%$ & $\mathbf{1 3 7 3 = 1 0 0 \%}$ \\
\hline
\end{tabular}


Grafik 3: Entwicklung Policeynormen Sexualdelikte / Ehe und Familie 19 Territorien

(Datengrundlage HÄRTER, STOLLEIS, Repertorium)

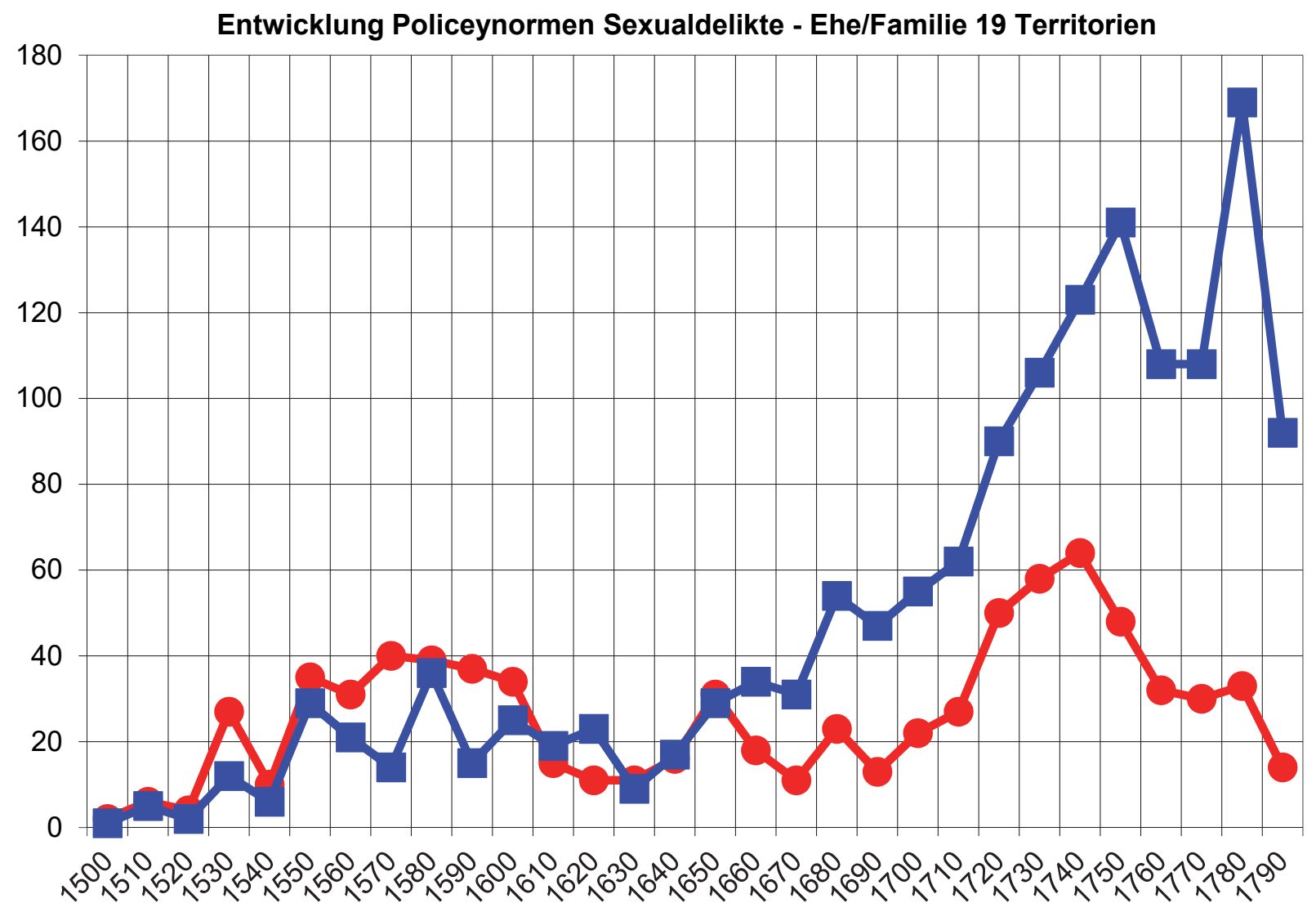




\section{Korrespondenz:}

Prof. Dr. Karl HÄRTER

Max-Planck-Institut für europäische Rechtsgeschichte Hansaallee 41

60323 Frankfurt am Main

haerter@rg.mpg.de

\section{Abkürzungen:}

Siehe das allgemeine Abkürzungsverzeichnis: [http://www.rechtsgeschichte.at/files/abk.pdf]

\section{Literatur:}

Gerhard AMMERER, Das Ende für Schwert und Galgen? Legislativer Prozess und öffentlicher Diskurs zur Reduzierung der Todesstrafe im Ordentlichen Verfahren unter Joseph II. 1781-1787 (Wien 2010).

Peter BECKER, Leben und Lieben in einem kalten Land. Sexualität im Spannungsfeld von Ökonomie und Demographie. Das Beispiel St. Lambrecht 1600-1850 (Frankfurt am Main 1990).

Peter BECKER, „Ich bin halt immer liederlich gewest und habe $\mathrm{zu}$ wenig gebetet". Illegitimität und Herrschaft im Ancien Régime, St. Lambrecht 1600-1850, in: Rudolf VIERHAUS (Hg.), Frühe Neuzeit - Frühe Moderne? Forschungen zur Vielschichtigkeit von Übergangsprozessen, (Göttingen 1992) 157-179.

Stefan BREIT, „Leichtfertigkeit" und ländliche Gesellschaft. Voreheliche Sexualität in der frühen Neuzeit (München 1991).

Susanna BURGHARTZ, Zeiten der Reinheit. Orte der Unzucht. Ehe und Sexualität in Basel während der Frühen Neuzeit (Paderborn 1999).

Egon Conrad ElLrichshausEn, Die uneheliche Mutterschaft im altösterreichischen Polizeirecht des 16. bis 18. Jahrhunderts dargestellt am Tatbestand der Fornication (Berlin 1988).

Wolfgang DOBRAS, Ratsregiment, Sittenpolizei und Kirchenzucht in der Reichsstadt Konstanz 15311548. Ein Beitrag zur Geschichte der oberdeutschschweizerischen Reformation (Gütersloh 1993).

Thomas DuvE, Was ist "Multinormativität“? Einführende Bemerkungen, in: Rechtsgeschichte 25 (2017) 88-101.

Ralf FRASSEK, Eherecht und Ehegerichtsbarkeit in der Reformationszeit. Der Aufbau neuer Rechtsstrukturen im sächsischen Raum unter besonderer Be- rücksichtigung der Wirkungsgeschichte des Wittenberger Konsistoriums (Tübingen 2005).

Ulrike GLEIXNER, „Das Mensch“ und „der Kerl“. Die Konstruktion von Geschlecht in Unzuchtsverfahren der Frühen Neuzeit (1700-1760) (Frankfurt am Main u. a. 1994)

Bettina GÜNTHER, Sittlichkeitsdelikte in den Policeyordnungen der Reichsstädte Frankfurt am Main und Nürnberg (15.-17. Jahrhundert), in: Karl HÄRTER (Hg.), Policey und frühneuzeitliche Gesellschaft (Frankfurt am Main 2000) 121-148.

Bettina GÜNTHER, Die Behandlung der Sittlichkeitsdelikte in den Policeyordnungen und der Spruchpraxis der Reichsstädte Frankfurt am Main und Nürnberg im 15. bis 17. Jahrhundert (Frankfurt am Main u. a. 2004)

André HolensteIn, Die Umstände der Normen - die Normen der Umstände. Policeyordnungen im kommunikativen Handeln von Verwaltung und lokaler Gesellschaft im Ancien Régime, in: KARLHÄRTER (Hg.), Policey und frühneuzeitliche Gesellschaft (Frankfurt am Main 2000) 1-46.

Beate HARMS-ZIEGLER, Illegitimität und Ehe. Illegitimität als Reflex des Ehediskurses in Preußen im 18. und 19. Jahrhundert (Berlin 1991).

Karl HÄRTER, Entwicklung und Funktion der Policeygesetzgebung des Heiligen Römischen Reiches Deutscher Nation im 16. Jahrhundert, in: Ius Commune 20 (1993) 61-141.

DERS., Fastnachtslustbarkeiten, Hochzeitsfeiern, Musikantenhalten und Kirchweih. Policey und Festkultur im frühneuzeitlichen Kurmainz, in: Mainzer Zeitschrift, Mittelrheinisches Jahrbuch für Archäologie, Kunst und Geschichte 92/93 (1997/98) 57-87.

DERS., Strafverfahren im frühneuzeitlichen Territorialstaat: Inquisition, Entscheidungsfindung, Supplikation, in: Andreas BlAUERT, Gerd SCHWERHOFF (Hgg.), Kriminalitätsgeschichte. Beiträge zur Sozial- und Kulturgeschichte der Vormoderne (Konstanz 2000) 459-480.

DERS., Kriminalität und Praxis der Strafjustiz im geistlichen Territorialstaat des Alten Reiches. Sexuelle Delinquenz und Justiznutzung im frühneuzeitlichen Kurmainz, in: Marco BellabARbA, Gerd SCHWERHOFF, Andrea ZORZI (Hgg.), Criminalità e giustizia in Germania e in Italia. Pratiche giudiziarie e linguaggi giuridici tra tardo Medioevo ed età moderna / Kriminalität und Justiz in Deutschland und Italien. Rechtspraktiken und gerichtliche Diskurse in Spätmittelalter und Früher Neuzeit (Bologna-Berlin 2001) 101-134.

DERS., Erfahrungen in der frühneuzeitlichen Strafjustiz, in: Paul MüNCH (Hg.), „Erfahrung” als Kate- 
gorie der Frühneuzeitgeschichte, (München 2001) 377-388.

DERS., Das Aushandeln von Sanktionen und Normen. $\mathrm{Zu}$ Funktion und Bedeutung von Supplikationen in der frühneuzeitlichen Strafjustiz, in: Cecilia NubOLA, Andreas Würgler (Hgg.), Bittschriften und Gravamina. Politik, Verwaltung und Justiz in Europa (14.-18. Jahrhundert) (Berlin 2005) 243274.

DERS., Policey und Strafjustiz in Kurmainz. Gesetzgebung, Normdurchsetzung und Sozialkontrolle im frühneuzeitlichen Territorialstaat (Frankfurt am Main 2005).

Ders., Polizei, in: Friedrich JAEger (Hg.), Enzyklopädie der Neuzeit Bd. 10 (Stuttgart 2009) 170-180.

DERS., Feuerbach, das Bayerische Strafgesetzbuch von 1813 und das Polizeistrafrecht, in: Arnd KоCH u.a., (Hgg.) Feuerbachs Bayerisches Strafgesetzbuch. Die Geburt liberalen, modernen und rationalen Strafrechts (Tübingen 2014) 129-147.

DERS., Kirchenzucht und gute Policey. Die Kirchenordnungen des 16. Jahrhunderts im Kontext der vormodernen Ordnungsgesetzgebung, in: Sabine AREND, Norbert HAAG, Sabine Holtz (Hgg.), Die württembergische Kirchenordnung von 1559 im Spannungsfeld von Religion, Politik und Gesellschaft (Epfendorf am Neckar 2013) 31-48.

DERS., Strafrechts- und Kriminalitätsgeschichte der Frühen Neuzeit (Berlin-Boston 2018).

DERS., Carl Theodor von Dalberg und das Strafrecht von den Reformen in Kurmainz bis zur Einführung des Code Pénal im Großherzogtum Frankfurt, in: Hans-Bernd SPIES (Hg.), Carl von Dalberg (1744-1817) und sein Umfeld (Aschaffenburg 2019).

DERS., Policeyordnungen, in: HRG² (Berlin 2018) 646652.

DERS., Policey, in: HRG² (Berlin 2018) 645-646

Ders., Michael STOLLEIS (Hgg.) Repertorium der Policeyordnungen der Frühen Neuzeit, Bd. 1-12 (Frankfurt am Main 1996-2017).

Susanne HEHENBERGER, Unkeusch wider die Natur. Sodomieprozesse im frühneuzeitlichen Österreich (Wien 2006).

Susanne HEHENBERGER, Sexualstrafrecht und Geschlechterordnung im frühneuzeitlichen Österreich, in: Gaby TeMME, Christine KüNZEL (Hgg.), Hat Strafrecht ein Geschlecht? Zur Deutung und Bedeutung der Kategorie Geschlecht in strafrechtlichen Diskursen vom 18. Jahrhundert bis heute, (Bielefeld 2010) 101-118.

Isabel V. Hull, Sexuality, State and Civil Society in Germany 1700-1815 (London 1996).
DIES., Sexualstrafrecht und geschlechtsspezifische Normen in den deutschen Staaten des 17. und 18. Jahrhunderts, in: Ute GERHARD (Hg.), Frauen in der Geschichte des Rechts. Von der frühen Neuzeit bis zur Gegenwart (München 1997) 221-234.

Claudia JARZEBOWSKI, Inzest. Verwandtschaft und Sexualität im 18. Jahrhundert (Köln u.a. 2006).

Vera JunG, Körperlust und Disziplin. Studien zur Fest- und Tanzkultur im 16. und 17. Jahrhundert, (Köln u. a. 2001).

Anke KELLER, Von verbotenen Feierfreuden. Hochzeits-, Tauf- und Begräbnisverordnungen im Frankfurt a. M. und Augsburg des 14. bis 16. Jahrhunderts (Heidelberg 2012).

Peter KLAMMER, In Unehren beschlaffen. Unzucht vor kirchlicher und weltlicher Gerichtsbarkeit im frühneuzeitlichen Salzburger Lungau, (Frankfurt am Main u. a. 2004).

Elisabeth $\mathrm{KOCH}$, Maior dignitas est in sexu virili. Das weibliche Geschlecht im Normensystem des 16. Jahrhunderts (Frankfurt am Main 1991).

Andreas MAISCH, „Unzucht“ und „Liederlichkeit“. Sozialdisziplinierung und Illegitimität im Württemberg der Frühneuzeit, in: Norbert HAAG, Sabine Holtz, Wolfgang ZimMERMAnNN (Hgg.), Ländliche Frömmigkeit. Konfessionskulturen und Lebenswelten 1500-1850 (Stuttgart 2002) 279-306.

Cecilia NubolA, Andreas Würgler, Suppliche e "gravamina". Politica, amministrazione, giustizia in Europa (secoli XV-XVIII) (Bologna 2002).

Christian PFISTER, Bevölkerungsgeschichte und historische Demographie 1500-1800 (München 1994)

August Ludwig REYSCHER (Hg.), Vollständige, historisch und kritisch bearbeitete Sammlung der württembergischen Gesetze, 19 Bde. (StuttgartTübingen 1828-1851).

Ulinka RuBLACK, Magd, Metz' oder Mörderin. Frauen vor frühneuzeitlichen Gerichten (Frankfurt am Main 1998).

Friedrich-Christian Schroeder (Hg.), Die peinliche Gerichtsordnung Kaiser Karls V. und des Heiligen Römischen Reichs von 1532 (Carolina) (Stuttgart 2000).

Dieter SCHWAB, Grundlagen und Gestalt der staatlichen Ehegesetzgebung in der Neuzeit bis zum Beginn des 19. Jahrhunderts (Bielefeld 1967).

Gerd SCHWERHOFF, Historische Kriminalitätsforschung (Frankfurt am Main 2011).

Uwe SIBETH, Eherecht und Staatsbildung. Ehegesetzgebung und Eherechtsprechung in der Landgrafschaft Hessen(-Kassel) in der frühen Neuzeit, (Darmstadt-Marburg 1994). 
Thomas SIMON, „Gute Policey“. Ordnungsleitbilder und Zielvorstellungen politischen Handelns in der frühen Neuzeit (Frankfurt am Main 2004).

Angela TAEGER, Moralstrafrecht und administrative Kontrolle der Sexualität im ausgehenden Ancien Régime (München 1999).

Otto UlBRICHT, Kindsmord und Aufklärung in Deutschland (München 1990).

Otto UlbRICHT (Hg.), Von Huren und Rabenmüttern. Weibliche Kriminalität in der Frühen Neuzeit, Köln u. a. 1995.

Rita VoltMER, Gegen die Unzucht. Nachtridentinische Sittenreform, Kriminalisierung und Verfolgung devianter Sexualität im Erzbistum Trier (16. und 17. Jahrhundert), in: Heinz-Günther
BORCK (Hg.), Unrecht und Recht. Kriminalität und Gesellschaft im Wandel von 1500-2000. Gemeinsame Landesausstellung der rheinlandpfälzischen und saarländischen Archive. Wissenschaftlicher Begleitband (Koblenz 2002) 481-511.

Matthias WEBER, Die Reichspolizeiordnungen von 1530, 1548 und 1577. Historische Einführung und Edition (Frankfurt am Main 2002).

Siegrid WestPHAL, Kirchenzucht als Ehe- und Sittenzucht. Die Auswirkungen von lutherischer Konfessionalisierung auf das Geschlechterverhältnis, in: Anne CONRAD (Hg.), „In Christo ist weder Man noch Weyb“. Frauen in der Zeit der Reformation und der katholischen Reform (Münster 1999) 152-171. 